\title{
Towards Understanding Ionic Transport Mechanisms of Sodium in Graphitic Materials by In Situ TEM
}

\author{
Kai $\mathrm{He}^{1,2}$
}

1. Department of Materials Science and Engineering, Northwestern University, Evanston, IL 60208, USA

2. NUANCE Center, Northwestern University, Evanston, IL 60208, USA

The graphitic materials are the most commonly used anode material for lithium-ion batteries. However, to meet the demand of more economical energy storage for large-scale applications, new battery chemistry is encouraged towards utilizing other alkali metals, specifically using sodium [1]. Although sharing many similarities with lithium counterparts, sodium ion batteries often show rather limited capacity for practical use, which was generally believed due to the larger ionic radius and so caused sluggish ionic diffusion and higher formation energy [2, 3]. Various efforts have been applied to engineering the graphitic materials' structures (soft/hard carbons) and morphologies (nanotubes, graphene, porous, hollow nanoparticles, etc.) to enable reversible sodium insertion into the graphitic interlayers and nanovoids. For example, we have demonstrated reversible sodiation and desodiation in reduced graphite oxides, where the interlayer spacing between adjacent graphene layers were tuned to $0.43 \mathrm{~nm}$ [4]. This is in a good agreement with theoretical calculations, which, however, also suggested due to a combination of energetic considerations rather than a sole effect of sodium size [5].

Here, we aim to clarify the relevant ambiguity towards understanding the electrochemical reactivity and ionic transport mechanisms of sodium in graphitic materials using in situ electron microscopy techniques. The in situ experiments were set up in the dry-cell configuration to allow for real-time imaging in TEM and STEM modes [2-4]. For simplicity, we use carbon nanotubes (CNTs) as a model material to perform a case study. Figure 1a shows a series of snapshot images during the in situ sodiation process, where the thickness of the CNT gradually increase as a function of time, indicating that sodium plating was occurred on the surface of CNT and eventually covered the entire nanotube, schematically shown in Figure 1c. For comparison, we prepared another sample by filling amorphous phosphor into the CNTs to form the bamboo-like structures, as shown in Figure 1b. The encapsulated phosphor nodes can be used as a clear indicator to assist us tracking the sodium transport. As shown in Figure $1 b$, the phosphor nodes react with sodium and exhibit distinct volume expansion as sodiation proceed as a function of time, while the thickness of the CNT only shows a slightly increase. This suggests a different ionic transport path: sodium ions shuffle through the multi-walls of the CNT with a higher speed comparing to surface plating, schematically shown in Figure 1d. The in situ observations show sodium transport in different paths, i.e. through surface diffusion or interlayer intercalation, which provides implications to future design and engineering of graphitic materials for sodium batteries [6].

\section{References:}

[1] MD Slater et al, Adv. Func. Mater. 23 (2013) 947.

[2] K He et al, ACS Nano 8, (2014) 7251.

[3] K He et al, Nano Lett. 15, (2015) 5755.

[4] Y Wen, K He et al, Nature Commun. 5, (2014) 4403. 
[5] Y Liu et al, Proc. Natl. Acad. Sci. 113, (2016) 3735.

[6] This work made use of the EPIC facility of Northwestern University's NUANCE Center, which has received support from the Soft and Hybrid Nanotechnology Experimental (SHyNE) Resource (NSF

ECCS-1542205); the MRSEC program (NSF DMR-1121262) at the Materials Research Center.
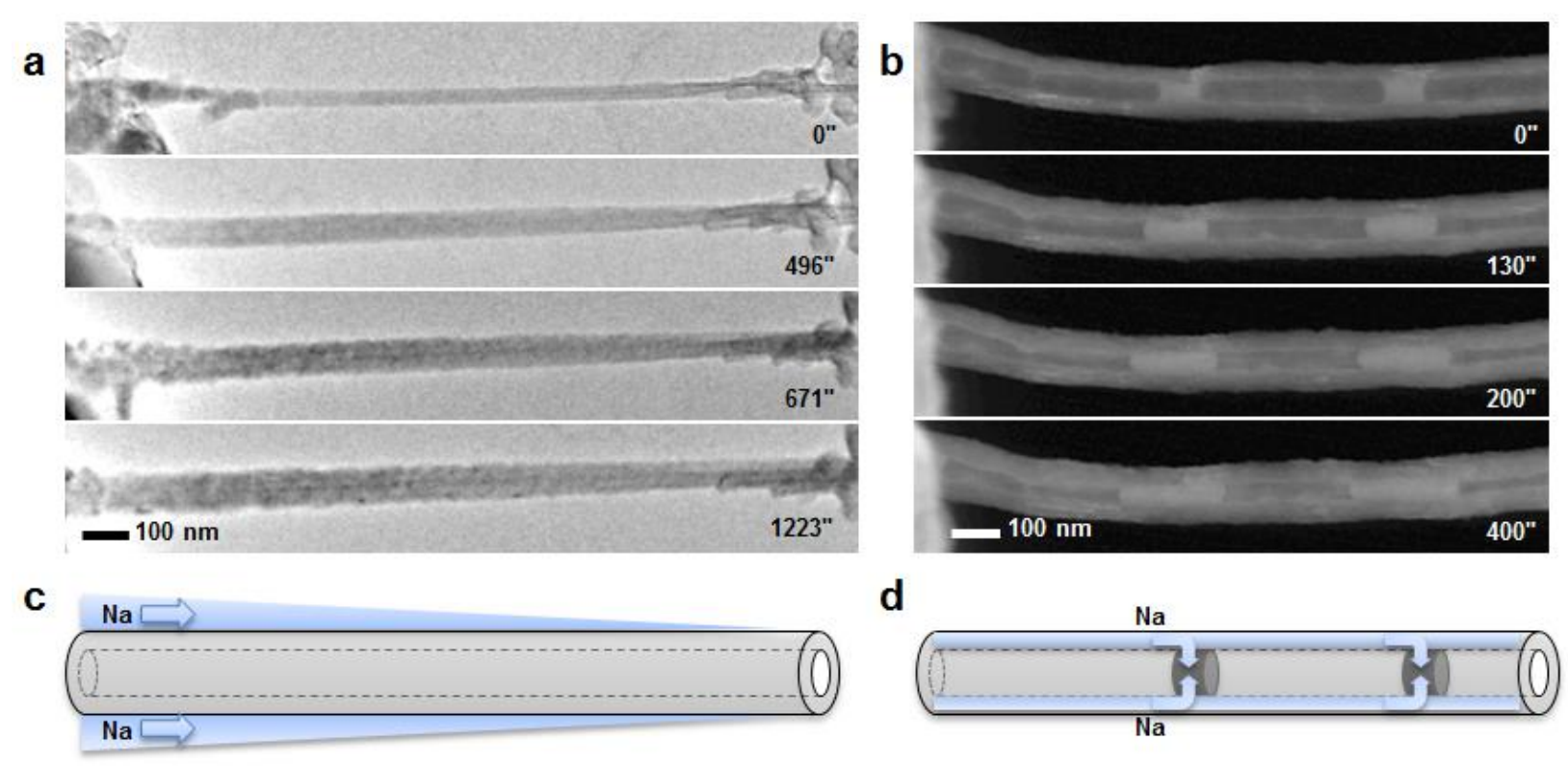

Figure 1. (a) In situ TEM images showing sodium plating on the surface of CNT. (b) In situ STEM images showing sodium intercalation through the CNT and reaction with the encapsulated phosphor nodes. Schematics showing sodium transport mechanisms of (c) surface diffusion, and (d) interlayer intercalation, respectively. 